\title{
Photon spectra from quark generation by WIMPs
}

\author{
J. A. R. Cembranos, A. Cruz-Dombriz ${ }^{1}$, A. Dobado, R. Lineros ${ }^{2}$ and A. L. Maroto \\ Departamento de Física Teórica I, Universidad Complutense de Madrid, E-28040 Madrid, Spain. \\ ${ }^{1}$ also at Department of Mathematics and Applied Mathematics, University of Cape Town, 7700 South Africa. \\ ${ }^{2}$ IFIC, CSIC-Universitat de Valencia, Ed. Instituts, Apt. 22085, 46071 Valencia, Spain.
}

\begin{abstract}
If the present dark matter (DM) in the Universe annihilates into Standard Model (SM) particles, it must contribute to the gamma ray fluxes that are detected on the Earth. The magnitude of such contribution depends on the particular DM candidate, but certain features of these spectra may be analyzed in a model-independent fashion. In this work we provide the fitting formula valid for the simulated photon spectra from WIMP annihilation into light quark-anti quark $(q \bar{q})$ channels in a wide range of WIMP masses. We illustrate our results for the $c \bar{c}$ channel.
\end{abstract}

Keywords: Dark matter, indirect searches, WIMPs, photon spectra and quark pairs annihilation.

PACS: $95.35 .+\mathrm{d}$ 98.80.Cq.

\section{INTRODUCTION}

According to present observations of large scale structures, $\mathrm{CMB}$ anisotropies and light nuclei abundances, DM cannot be accommodated within the SM of elementary particles. Indeed, DM presence is a required component on cosmological scales, but also to provide a satisfactory description of rotational speeds of galaxies, orbital velocities of galaxies in clusters, gravitational lensing of background objects by galaxy clusters and the temperature distribution of hot gas in galaxies and clusters of galaxies. The experimental determination of the DM nature will require the interplay of collider experiments and astrophysical observations. These searches use to be classified in direct or indirect searches (see [1] and references in Introduction in [2]) . Concerning direct ones, the elastic scattering of DM particles from nuclei should lead directly to observable nuclear recoil signatures although the weak interactions between DM and the standard matter makes DM direct detection extremely difficult.

On the other hand, DM might be detected indirectly, by observing their annihilation products into standard model particles. Thus, even if WIMPs (Weakly Interacting Massive Particles) are stable, two of them may annihilate into ordinary matter such as quarks, leptons and gauge bosons. Their annihilation in different places (galactic halo, Sun, etc.) produce cosmic rays to be discriminated through distinctive signatures from the background. After WIMPs annihilation a cascade process occurs. In the end the stable particles: neutrinos, gamma rays, antimatter... may be observed through different devices. Neutrinos and gamma rays have the advantage of maintaining their original direction due to their null electric charges.
This communication precisely focuses on photon production coming from $q \bar{q}$ channels (except $t \bar{t}$ channel). Photon fluxes in specific DM models are usually obtained by software packages such as DarkSUSY and micrOMEGAs based on PYTHIA Monte Carlo event generator [3] after having fixed a WIMP mass for the particular SUSY model under consideration. In this sense, the aim of this investigation is to provide fitting functions for the photon spectra corresponding to each individual annihilation $q \bar{q}$ channel and, in addition, determine the dependence of such spectra on the WIMP mass in a model independent way. This would allow to apply the results to alternative DM candidates for which software packages have not been developed. On the other hand, the information about channel contribution and mass dependence can be very useful in order to identify gamma-ray signals for specific WIMP candidates and may also provide relevant information about the photon energy distribution when $q \bar{q}$ pairs annihilate.

Let us remind that the $\gamma$-ray flux from the annihilation of two WIMPs of mass $M$ into two SM particles coming from all possible annihilation channels (labelled by the subindex $i$ ) is given by:

$$
\begin{aligned}
\frac{\mathrm{d} \Phi_{\gamma}^{\mathrm{DM}}}{\mathrm{d} E_{\gamma}}= & \frac{1}{4 \pi M^{2}} \sum_{i}\left\langle\sigma_{i} v\right\rangle \frac{\mathrm{d} N_{\gamma}^{i}}{\mathrm{~d} E_{\gamma}} \\
& \times \frac{1}{\Delta \Omega} \int_{\Delta \Omega} \mathrm{d} \Omega \int_{\text {l.o.s. }} \rho^{2}[r(s)] \mathrm{d} s,
\end{aligned}
$$

where $\left\langle\sigma_{i} v\right\rangle$ holds for the thermal averaged annihilation cross-section of two WIMPs into two ( $i^{\text {th }}$ channel) SM particles and $\rho$ is the DM density. The integral is performed along the line of sight (l.o.s.) to the target and averaged over the detector solid angle $\Delta \Omega$. 
TABLE 1. $b_{1}, n_{1}, c_{1}, d_{1}$ and $p$ parameters corresponding to expression (2) in the $c \bar{c}$ channel. Mass independent parameters in (2) for this channel are $a_{1}=5.58$; $b_{2}=7.90 ; n_{2}=0.686 ; c_{2}=0.0 ; q=9.00 \cdot 10^{-4}$.

\begin{tabular}{rrrrrr}
\hline$M(\mathbf{G e V})$ & $b_{1}$ & $n_{1}$ & $c_{1}$ & $d_{1}$ & $p$ \\
\hline 50 & 5.93 & 2.35 & 0.239 & 0.428 & 210 \\
100 & 5.48 & 2.08 & 0.283 & 0.374 & 379 \\
200 & 4.98 & 1.86 & 0.330 & 0.330 & 673 \\
500 & 4.50 & 1.65 & 0.378 & 0.288 & 1230 \\
1000 & 4.00 & 1.50 & 0.406 & 0.264 & 2110 \\
2000 & 3.70 & 1.35 & 0.432 & 0.245 & 4050 \\
5000 & 3.27 & 1.17 & 0.470 & 0.221 & 8080 \\
8000 & 3.08 & 1.11 & 0.494 & 0.208 & 12000 \\
\hline
\end{tabular}

\section{PROCEDURE}

We have used the particle physics PYTHIA software [3] to obtain our results. The WIMP annihilation is usually splited into two separated processes: The first describes the annihilation of WIMPs and its SM output. The second one considers the evolution of the obtained SM unstable products. Due to the expected velocity dispersion of DM, we expect most of the annihilations to happen quasi-statically. This fact allows to state that by considering different center of mass $(C M)$ energies for the obtained SM particles pairs from WIMP annihilation process, we are indeed studying different WIMP masses, i.e. $E_{\mathrm{CM}} \simeq 2 M$. The procedure to obtain the photon spectra is thus straightforward: For a given pair of SM particles which are produced in the WIMP annihilation, we count the number of photons in bins for the variable $x \equiv E_{\gamma} / M$.

Once the PYTHIA simulations have been performed, the required parametrization to fit the data for the $q \bar{q}$ channels (except $t \bar{t}$ ) may be written as:

$$
\begin{aligned}
& \frac{\mathrm{d} N_{\gamma}}{\mathrm{d} x}=\frac{a_{1}}{x^{1.5}} \exp \left(-b_{1} x^{n_{1}}-b_{2} x^{n_{2}}-\frac{c_{1}}{x^{d_{1}}}+\frac{c_{2}}{x^{d_{2}}}\right) \\
+\quad & q \ln [p(1-x)] \frac{x^{2}-2 x+2}{x}
\end{aligned}
$$

The parameters in expression (2) were considered to be WIMP mass dependent. After a fitting process they were determined for different WIMP masses, in a range varying from 50 to 7000 (or 8000 ) GeV. Mass dependences for the parameters in (2) were fitted by using power laws.

\section{III. $c \bar{c}$ CHANNEL}

In order to illustrate the explained procedure, we study the $c \bar{c}$ channel. For this channel there are five mass dependent parameters in expression (2): $b_{1}, n_{1}, c_{1}, d_{1}$ and $p$ presented in Table I. The mass independent parameters are $a_{1}, b_{2}, n_{2}, c_{2}\left(d_{2}\right.$ is thus irrelevant) and $q$. In Table
TABLE 2. Fitting power laws in $c \bar{c}$ channel.

\begin{tabular}{rrr}
\hline Parameter & Interval $(\mathbf{G e V})$ & Power law(s) \\
\hline$b_{1}$ & $50 \leq M \leq 8000$ & $9.90 M^{-0.130}$ \\
$n_{1}$ & $50 \leq M \leq 8000$ & $4.14 M^{-0.148}$ \\
$c_{1}$ & $500 \leq M \leq 8000$ & $0.210 M^{0.0951}$ \\
$d_{1}$ & $50 \leq M \leq 8000$ & $1.50 M^{-0.632}$ \\
& & $+0.479 M^{-0.0942}$ \\
$p$ & $200<M \leq 8000$ & $8.11 M^{0.812}$ \\
\hline
\end{tabular}

II we present the fitting power laws for mass dependent parameters. Figure 1 presents spectra for four different WIMP masses whereas Figure 2 shows fitting power laws for two mass dependent parameters. The results for the other $q \bar{q}$ channels [2] are completely analogous even though for each channel, the parameters which are mass dependent may be different to the ones for the $c \bar{c}$ channel.

\section{CONCLUSIONS}

In this work, we have studied the photon spectra coming from WIMP pair annihilation into $q \bar{q}$ pairs for all the channels (except $t \bar{t}$ ). The covered WIMP mass range was from $50 \mathrm{GeV}$ to $8000 \mathrm{GeV}$. Simulated spectra covered the whole accessible energy interval: from extremely low energetic photons till photons with one half of the available total center of mass energy.

Once the spectra were simulated, an analytical expression (2) was proposed to fit the data. This expression depends on both WIMP mass dependent and independent parameters. Our results can both provide a better understanding of the DM annihilation channels into photons and save an important amount of unnecessary Monte Carlo simulations. This fact is particularly important for high energy photons, whose production rate is very suppressed.

Calculations for all $q \bar{q}$ channels are available at the website http://teorica.fis.ucm. es/ PaginaWeb/downloads.html. This work was partially supported by MULTIDARK CSD200900064.

\section{REFERENCES}

1. K. Sigurdson and M. Kamionkowski, PRL 92, 171302 (2004); J. A. R. Cembranos et al. , PRL 90, 241301 (2003); J. A. R. Cembranos and L. E. Strigari, PRD 77, 123519 (2008); J. A. R. Cembranos, PRL 102, 141301 (2009).

2. J. A. R. Cembranos, A. de la Cruz-Dombriz, A. Dobado, R. A. Lineros, A. L. Maroto, arXiv: hep-ph/1009.4936.

3. T. Sjostrand, S. Mrenna and P. Skands, JHEP05 (2006) 026 (LUTP 06-13, FERMILAB-PUB-06-052-CD-T). 

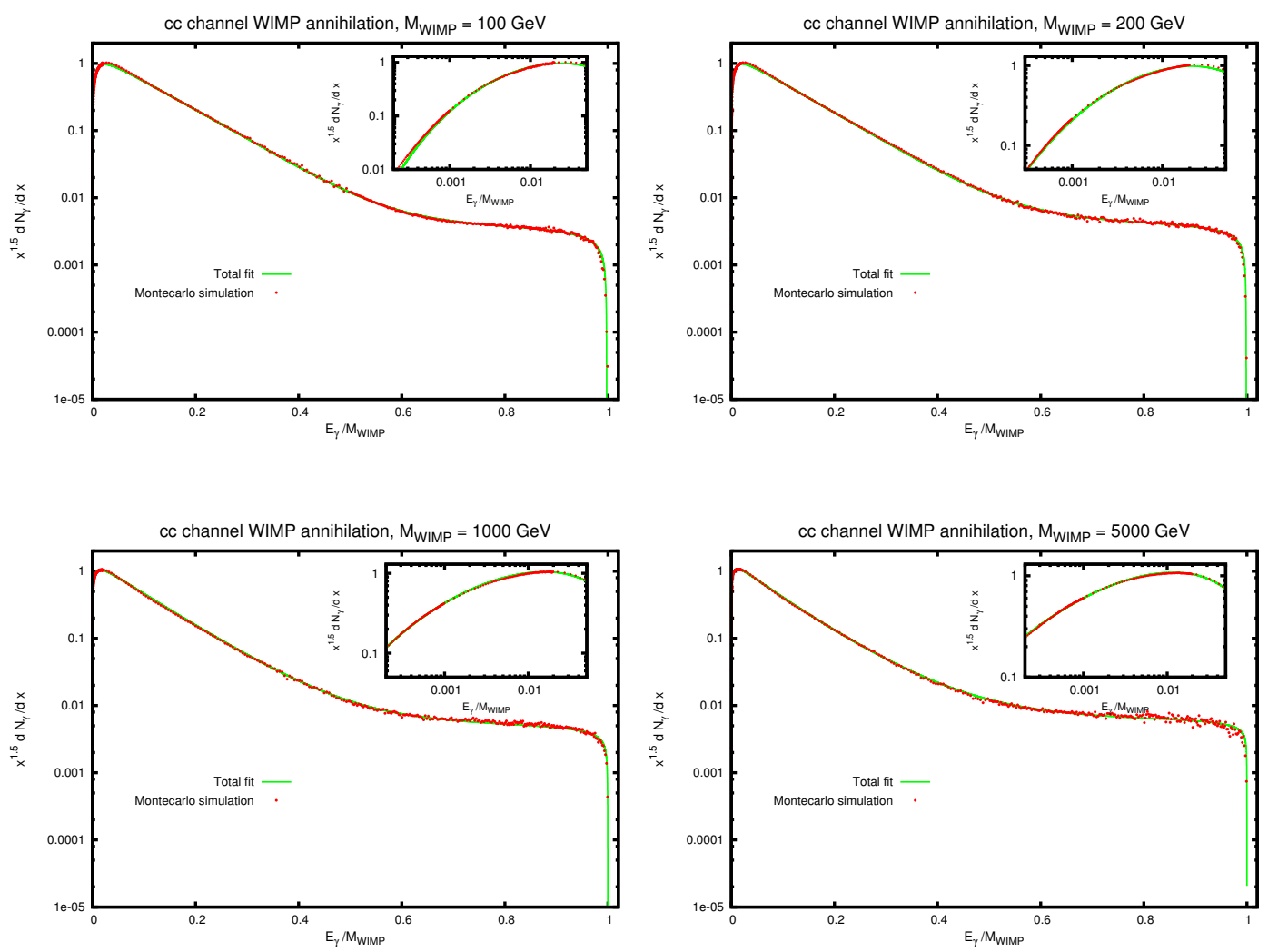

FIGURE 1. Photon spectra for four different WIMP masses $(100,200,1000$ and $5000 \mathrm{GeV})$ in the $c \bar{c}$ annihilation channel. Red dotted points are PYTHIA simulations and solid lines correspond to the proposed fitting functions.
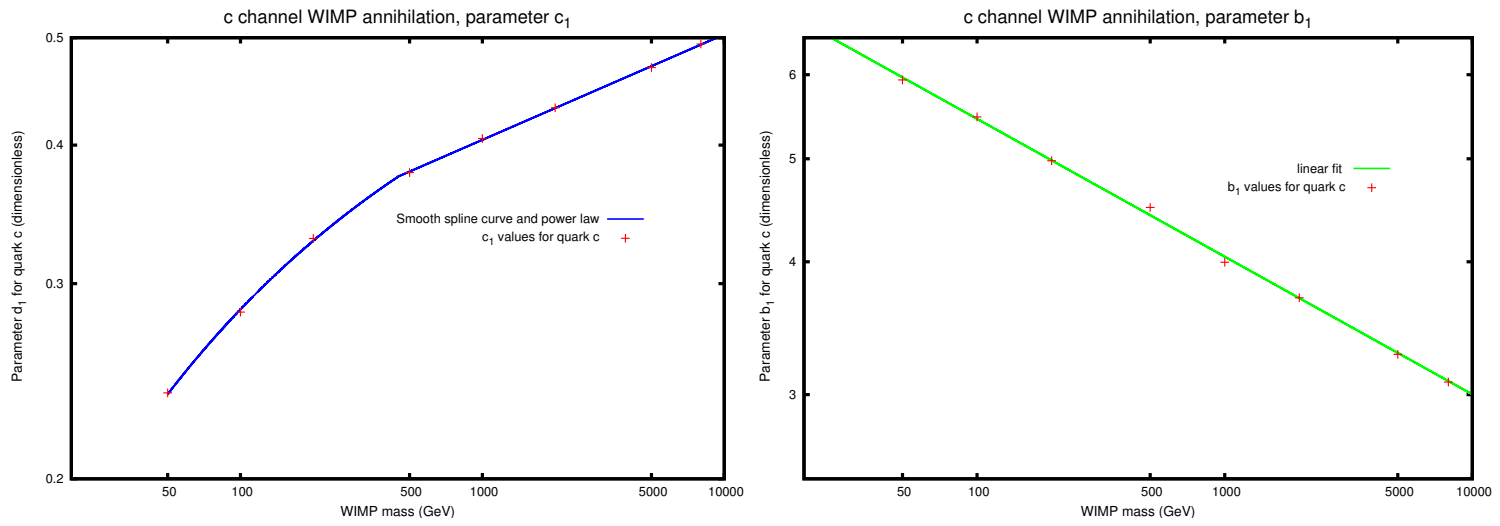

FIGURE 2. Mass dependence of $c_{1}$ and $b_{1}$ parameters for $c \bar{c}$ annihilation channel. Crossed points are parameters values found after the fitting process for each WIMP mass and solid lines correspond to the proposed fitting functions. 
Copyright of AIP Conference Proceedings is the property of American Institute of Physics and its content may not be copied or emailed to multiple sites or posted to a listserv without the copyright holder's express written permission. However, users may print, download, or email articles for individual use. 